\section{Overall survival of adult acute myeloid leukemia based on cytogenetic and molecular abnormalities during 5 years in a single center study}

\author{
Enaam M. Alsobhi, FRCPI, FRCPath, \\ Fayssal M. Farahat, MD, PhD, \\ Mustafa F. Daghistani, PhD, MSc, \\ Khadeeja A. Awad, Medical Student, \\ Omar S. Al-Zahrani, Medical Student, \\ Afaf S. Al-Saiari, Medical Student, \\ Fai K. Koshak, Medical Student.
}

\begin{abstract}
Objectives: To determined the 5-year overall survival (OS) rates for adult patients with acute myeloid leukemia (AML) patients at King Abdulaziz Medical City, Jeddah, Saudi Arabia, based on cytogenetic and molecular abnormalities.
\end{abstract}

Methods: A retrospective cohort study reviewing adult AML patient files between 2011 and 2018. Sixty-three patients were excluded due to pediatric age and secondary AML. The remaining 87 adult patients with de novo AML were enrolled in this study.

Results: The most frequent cytogenetic abnormalities were $\mathrm{t}(15 ; 17) \quad(17.2 \%)$, followed by complex cytogenetic $(13.8 \%)$ and $t(8 ; 21)(5.7 \%)$. The most frequent molecular abnormalities were promyelocytic leukemia/retinoic acid receptor alpha (PML-RARA) (16.1\%) and Nucleophosmin 1 (NPM1) (11.5\%). The highest OS rate was associated with $t(15 ; 17)$, PML-RARA, and NPM. However, complex cytogenetic was associated with the lowest OS rate; fms-like tyrosine kinase 3 (FLT3)-internal tandem duplication was independently correlated with low OS rate.

Conclusion: The study describes cytogenetic and molecular abnormalities observed in adult AML patients and gives an overview of prognostic factors and determine the OS, with comparable results with recent published data by the WHO.

\section{Saudi Med J 2019; Vol. 40 (11): 1171-1176} doi: 10.15537/smj.2019.11.24584

cute myeloid leukemia (AML) is caused by the abnormal A proliferation of myeloblasts in bone marrow. ${ }^{1,2}$ In 2012, the worldwide incidence of AML was 4.7 per 100,000 people and the worldwide mortality rate were 3.4 per 100,000 people. ${ }^{3}$ In 2014,693 cases of adult acute leukemia, which represented $5.9 \%$ of all cancer types, were reported by the Saudi Cancer Registry; of these, 304 were AML cases, ${ }^{4}$ According to the World Health Organization (WHO), the 5-year survival rate for adult patients with AML is approximately $40 \% .^{5}$ Acute myeloid leukemia is a heterogeneous group of disorders caused by different gene mutations and cytogenetic abnormalities, which consequently have various clinical symptoms and outcomes, and are a key prognostic determinant in AML. ${ }^{6}$ World Health Organization ${ }^{5}$ classifies the prognosis of AML into good, intermediate, or poor categories. AML associated with chromosomal rearrangements such as $\mathrm{t}(15 ; 17)$-promyelocytic leukemia/retinoic acid receptor alpha (PML-RARA), inv (16), $\mathrm{t}(8 ; 21), \mathrm{t}(16 ; 16)$, and mutated Nucleophosmin 1 (NPM1) with no FLT3-ITD has a relatively favorable prognosis, whereas the presence of $+8,+21$, and +22 is associated with an intermediate prognosis. However, monosomy 5 or 7, fms-like tyrosine kinase 3 (FLT3)-internal tandem duplication (ITD), and c-KIT mutations are associated with a poor prognosis. ${ }^{5}$ Secondary AML is usually associated with multiple cytogenetic abnormalities and has a poor outcome. ${ }^{7}$ We made an extended literature review; however, previous studies are very limited in Saudi Arabia or other Arab country in predicting the cumulative 5 years overall survival (OS) of adult patients with AML based on molecular and cytogenetic classifications. Therefore, we propose for a retrospective study at King Abdulaziz Medical City (KAMC), Jeddah, Saudi Arabia to evaluate these abnormalities associated with OS.

Methods. This retrospective cohort study took place at KAMC, Jeddah, Saudi Arabia, reviewed 150 adult patient files between May 2011 and May 2018. Of these, 63 cases were excluded; 39 due to secondary AML and 24 were children. Eighty-seven patients having age more than 18 years with de novo AML were included in this study. The OS rate was calculated to identify OS according to the presence of specific gene factors. Most patients diagnosed from 2011 to 2018 were Saudi, except one Yemeni patient. Data collected included age, gender, nationality, type of AML, clinical presentation at the time of diagnosis, blood test results, bone marrow result, flow cytometry, cytogenetic/molecular results, date of diagnosis, prognosis, patient status, and cause of death. The Helsinki Declaration guidelines for ethical principles in medical research were applied to this study. All patients in this study had received the standard ' $7+3$ ' regimen ( 7 days of cytosine arabinoside with 3 days of anthracycline). In addition, promyelocytic leukemia (M3) patients were treated with the same standard regimen plus all-trans retinoic acid (ATRA). The Research Ethics Committee at King Abdullah 
International Medical Research Center (KAIMARC) approved this study (Study No. RSS18/36/J).

Cytogenetic analysis. Chromosomal analysis was performed on overnight culture samples of unstimulated bone marrow (BM) using standard procedures. TrypsinGiemsa banding was performed and 20 metaphase chromosomes were karyotyped using CytoVision v. 7.4 software (Biosystems, Wetzlar, Germany). Karyotypes were defined based on the International System for Human Cytogenetic Nomenclature (ISCN-2016).

Fluorescence in situ hybridization (FISH). Fluorescence in situ hybridization was performed using BM samples and the AML FISH panel to identify the presence of abnormalities commonly observed in leukemic cells that did not divide in culture. Visys FISH probes were procured from Abbott Laboratories (Abbott Park, USA). In total, 100 interphase cells were analyzed. Tests were performed at Bio Scientia Laboratories (ingelheim, Rheinland-pfalz Germany) and reviewed by the Cytogenetics Unit, KAMC. The results of FISH testing were used for investigational purposes only. Acute myeloid leukemia FISH Panel: $\mathrm{t}(15 ; 17)(\mathrm{q} 24 ; \mathrm{q} 21)$ PML-RARA; $\mathrm{t}(8 ; 21)$ (q22;q22) RUNX1T1-RUNX1 (ETO-AML1); inv(16)(p13.3q22) CBFB; 11q23 KMT2A (MLL); inv(3) or t(3;3) RPN1-MECOM (EVI1); del(5)(q31) EGR1; del(7)(q31)/-7 D7S486; $+8 ; \operatorname{del}(20 \mathrm{q})$

Mutation analysis. Mutation analyses were performed at Bio Scientia Laboratories and reviewed by the Cytogenetics Unit, KAMC. The results of mutation analysis were used for investigational purposes only. The AML panel included NPM1, CEBPA, and c-KIT FLT3-ITD mutations and was used to define the incidence of abnormalities frequently detected in AML with normal chromosome analysis.

Statistical analysis. Data were analyzed using IBM SPSS v. 23 (IBM Corp., Armonk, USA). The Shapiro-Wilk test was used to determine the normality of the distribution of continuous variables. Variables were presented using mean, standard deviation, and median for normally and non-normally distributed continuous variables; plain number and percentage were used for categorical variables. The Kaplan-Meier survival curve using the log rank test was performed to

Disclosure. Authors have no conflict of interests, and the work was not supported or funded by any drug company. identify survival of cases according to the presence of specific gene factors. The magnitude of the effect was determined using Cox regression and hazard ratio with a $95 \%$ confidence interval. The level of significance was determined at $p<0.05$.

Results. The most frequent laboratory parameters of the patients are summarized in Table 1 . The FrenchAmerican-British (FAB) classification of AML subtypes

Table 1 - Clinical and epidemiological characteristics of the 87 patients included in the study.

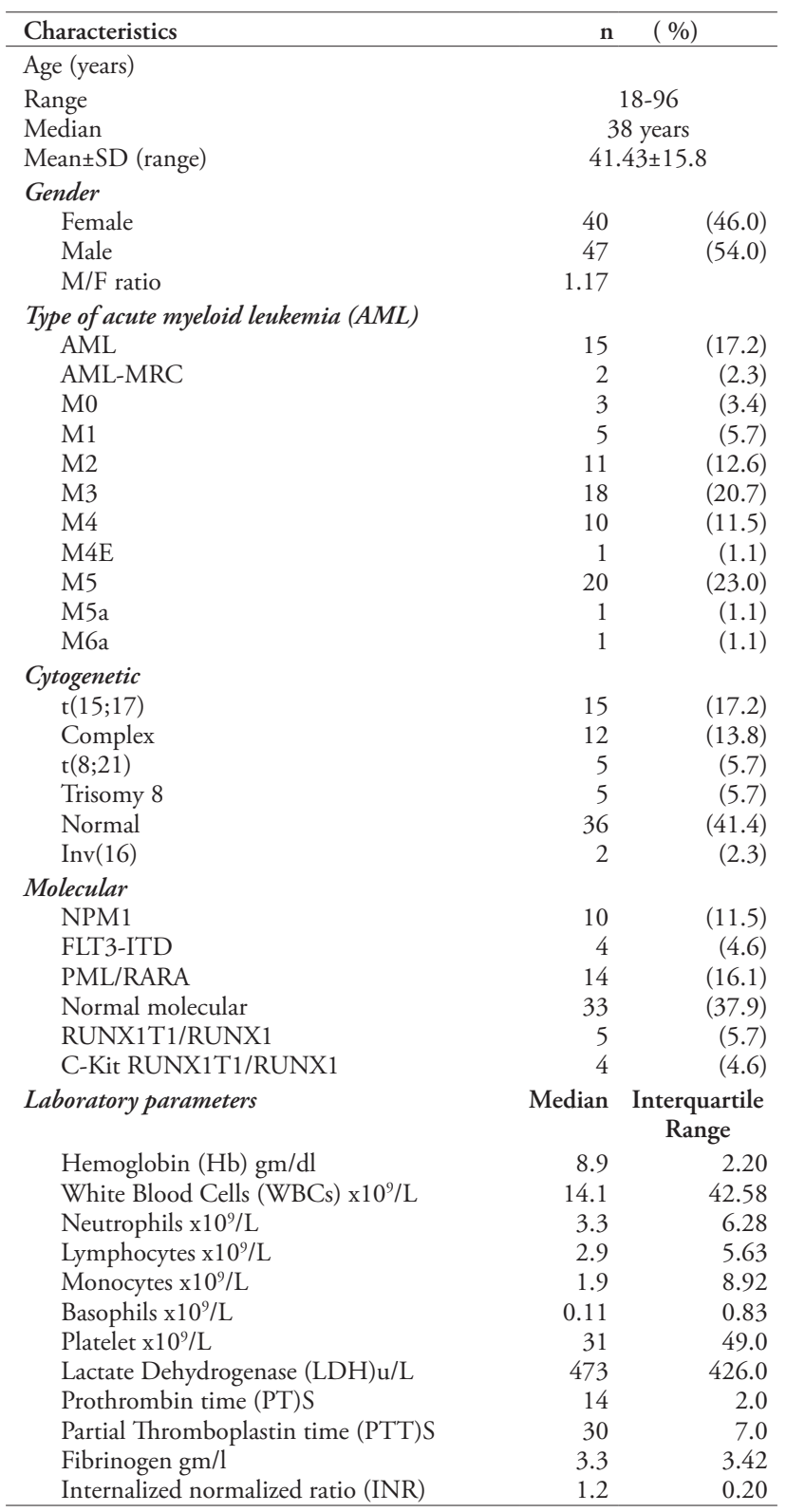



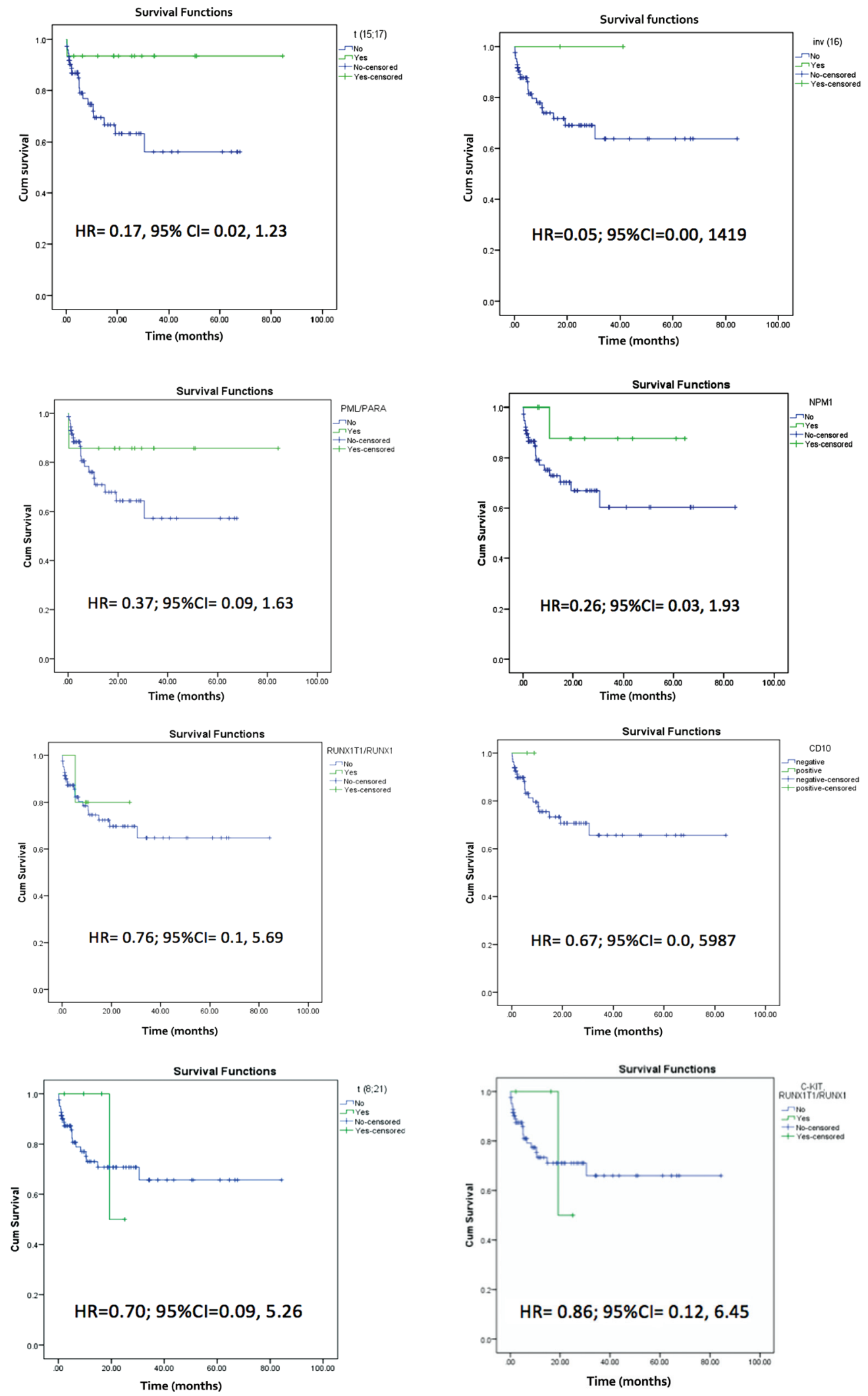

Figure 1 - Kaplan-Meier curve and Cox-regression analysis of genes associated with better prognosis. 

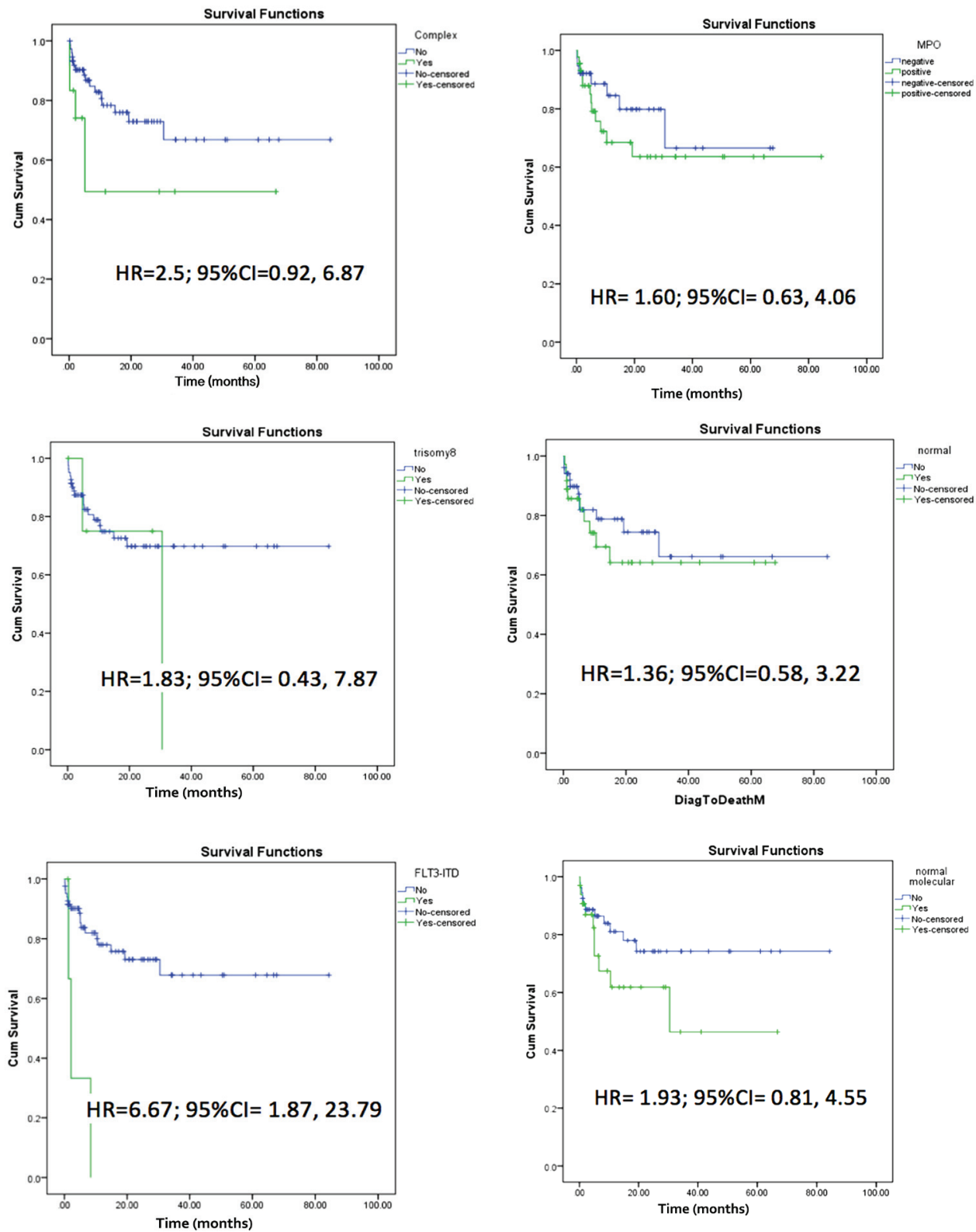

Figure 2 - Kaplan-Meier curve and Cox-regression analysis of factors with poor prognosis.

was applied to this study, except for 15 patients $(17.2 \%)$ whose details were not available. In this study, M5 (24.1\%) was found to be the most common FAB subtype, followed by M3 (20.7\%), M4 (12.6\%), M2 (12.6\%), M1 (5.7\%), M0 (3.4\%), and M6 (1.1\%).

Cytogenetic and molecular analysis. Patients were found to have cytogenetic and molecular variations, as shown in Table 1. Figure 1 depicts survival curves of factors associated with better prognosis as determined by the hazard ratio, HR). Although the presence of $\mathrm{t}(15 ; 17)$, inv(16), NPM1, PML/PARA, C-kit
RUNX1T1/RUNX1, and $t(8 ; 21)$ indicated better survival, none of these genes and factors showed statistically significant differences. Based on HR, the genes and factors associated with poor prognosis included complex, trisomy 8, normal cytogenetic, FLT3-ITD, normal molecular, MPO, CD56, CD34, HLADR, CD7 MPO, CD56, CD34, HLADR, and CD7 (Figure 2). However, none of the differences were statistically significant.

Clinical features of AML patients. In this study, the most frequent clinical presentation at the time of 
diagnosis was fever, followed by fatigue. Specifically, 45 cases presented with fever $(52.7 \%), 32$ with fatigue (36.8\%), 17 with bleeding (19.5\%), 15 with bone ache (17.2\%), 11 with weight loss (12.6\%), 9 with petechiae and ecchymosis (10.3\%), 7 with bruises (8\%), 4 with splenomegaly (4.6\%), 3 with lymphadenopathy $(3.4 \%)$, and 1 with hepatomegaly (1.1\%).

Discussion. Although studies report a prognostic relationship with cytogenetic and molecular abnormalities in adult AML, ${ }^{6,8}$ improving the OS of AML patients remains a challenge. Only WHO has reported OS for adult AML during the last 15 years which we will discuss later in comparison with current study. ${ }^{5}$ This study assessed a group of adults in Saudi Arabia with AML characterized by various cytogenetic and molecular aberrations (Table 1), which were examined for their prognostic value on the OS. Promyelocytic leukemia (PML) used to be fatal during the last 3 decades; however, has shown remarkable improvement in OS compared to other types of AML.? In 2017, $\mathrm{Ng} \& \mathrm{Chng}^{10}$ reported that OS was enhanced by $82 \%$ when ATRA was added to the standard therapy. However, the same study reported that patients with FLT3-ITD associated with PML seemed to have an inferior OS at 3 years $(62 \%$ OS).

Alrajeh et $\mathrm{al}^{11}$ reported that the most frequent cytogenetic aberration in 100 bone marrow cases reviewed in Riyadh, Saudi Arabia was trisomy 8, followed by $\mathrm{t}(8 ; 21)$; while the most frequent molecular abnormality was NPM1, followed by FLT3-ITD. In contrast, the results of this study found that $\mathrm{t}(15 ; 17)$ was the most frequent cytogenetic abnormality, followed by complex cytogenetics; while the most frequent molecular abnormality was PML/RARA, followed by NPM1. These differences could be due to the small sample size of this study or to differing epidemiology of AML in different regions of Saudi Arabia, in addition to median age, and when the studies were conducted. Manola et $\mathrm{al}^{12}$ found the M2 FAB classification most frequently, followed by M3. In the current study, M5 was the most frequent FAB subtype, followed by M3. However, the cytogenetic abnormalities $\mathrm{t}(15 ; 17), \mathrm{t}(8 ; 21)$, and inv(16) were associated with favorable prognosis. Neither study results correlated with OS. ${ }^{11,12}$ The 2017 WHO study reported that $\mathrm{t}(15 ; 17)$ was associated with high $81 \%$ OS, $t(8 ; 21)$ with $61 \%$ OS, and inv(16) with $55 \%$ OS; ${ }^{5}$ complex cytogenetics was associated with an unfavorable prognosis. However, Byrd et $\mathrm{al}^{13}$ reported OS rates in 2002 for 1213 adult patients with AML in 3 prognosis groups: favorable $(55 \%)$, intermediate
(24\%), and adverse (5\%). The results of our study concurred with their results (Figures $1 \& 2$ ).

The molecular abnormalities, NPM1, PML-RARA, and RUNX1T1/RUNX1 were accompanied with favorable prognosis in the WHO study, ${ }^{5}$ which concurred with RUNX1T1/RUNX1 associated with good prognosis in the current study. However, our results are disagreeing in contrast to those reported by Velloso et al.?

In conclusion, the current study describes different cytogenetic and molecular abnormalities detected in adult AML in single center, Saudi population and gives an overview in predicting the prognosis of the disease, which is agreeable with that previously reported by WHO. However, no significant changes in OS in the last 10 years for AML type except for AML-M3. The challenge in improving the OS probably depend on future therapy yet to be answered

There are some limitations to this study as it is a retrospective study, in a single center with limited population size. The study has the chances of bias inherent to retrospective design, and subgroup analyses were not possible due to the small number of subjects in some categories. Therefore, future multicenter studies with similar inclusion and exclusion criteria are necessary to validate the results of this study.

Acknowledgment. The authors would like to thank the medical record and laboratory staff at King Abdulaziz Medical City, Jeddah, Saudi Arabia for their assistance. Also, we would like to thank the Editage (https://www.editage.com) for English language editing.

Received 14th June 2019. Accepted 17th September 2019.

From the Abdulaziz Medical City, King Saud University Medical College and King Abdullah International Medical Research Center (Al-Sobhi, Farahat, Daghistani); from the College of Medicine (Awad, Al-Zahrani, Alsaiari), King Abdulaziz University; and from Al-Batterjee Medical College (Koshak), Jeddah, Kingdom of Saudi Arabia.

Address correspondence and reprints request to: Dr. Enaam Al-Sobhi, Consultant Hematology, Assistant Professor, King Abdulaziz Medical City, King Saud University, and King Abdullah International Medical Research Center, Jeddah, Kingdom of Saudi Arabia. E-mail:ensobhi@gmail.com

ORCID ID: https://orcid.org/0000-0002-5592-2921

\section{References}

1. Dohner H, Weisdorf DJ, Bloomfield CD. Acute myeloid leukemia. N Engl J Med 2015; 373: 1136-1152.

2. American Cancer Society. What is acute myeloid leukemia; Atlanta: American Cancer Society; 2018.

3. Ferlay J, Soerjomataram I, Ervik M, Dikshit R, Eser S, Mathers C, et al. GLOBOCAN 2012 v1.0, Cancer Incidence and Mortality Worldwide: IARC CancerBase No. 11 [Internet]. Lyon (FR): International Agency for Research on Cancer; 2013. 
4. Saudi Cancer Registry. Cancer Incidence Report in Saudi Arabia 2014-2017. Riyadh (KSA): Saudi Cancer Registry; 2017.

5. World Health Organization. AML classification biology and prognosis. Geneva: World Health Organization; 2017.

6. Cancer and Leukemia Group B 84611, Farag SS, Archer KJ, Mrózek K, Ruppert AS, Carroll AJ, et al. Pretreatment cytogenetics add to other prognostic factors predicting complete remission and long-term outcome in patients 60 years of age or older with acute myeloid leukemia: results from Cancer and Leukemia Group B 8461. Blood 2006; 108: 63-73.

7. Velloso ED, Motta CH, Furtado JB, Bacal NS, Silveira PA, Moyses CB, et al. Molecular and cytogenetic abnormalities in acute myeloid leukemia: and case studies. Einstein (Sao Paulo) 2011; 9: 184-189.

8. Gupta M, Mahapatra M, Saxena R. Cytogenetics' impact on the prognosis of acute myeloid leukemia. J Lab Physicians 2019; 11: 133.
9. Wang ZY, Chen Z. Acute promyelocytic leukemia: from highly fatal to highly curable. Blood 2008; 111: 2505-2515.

10. Ng C, Chng W. Recent advances in acute promyelocytic leukaemia. F1000Res 2017; 6: 1273.

11. Alrajeh A, Abalkhail H, Khalil S. Cytogenetics and molecular markers of acute myeloid leukemia from a tertiary care center in Saudi Arabia. Journal of Applied Hematolology 2017; 8: 68-74.

12. Manola KN, Panitsas F, Polychronopoulou S, Daraki A, Karakosta M, Stavropoulou C, et al. Cytogenetic abnormalities and monosomal karyotypes in children and adolescents with acute myeloid leukemia: correlations with clinical characteristics and outcome. Cancer Genet 2013; 206: 63-72.

13. Byrd JC, Mrózek K, Dodge RK, Carroll AJ, Edwards CG, Arthur DC, et al. Pretreatment cytogenetic abnormalities are predictive of induction success, cumulative incidence of relapse, and overall survival in adult patients with de novo acute myeloid leukemia: results from Cancer and Leukemia Group B (CALGB 8461). Blood 2002; 100: 4325-4336. 